Indian J. Genet., 75(1): 57-61 (2015)

DOI: 10.5958/0975-6906.2015.00006.1

\title{
Molecular diversity based heterotic groups in pigeonpea [Cajanus cajan (L.) Millsp.]
}

\author{
Bharathi Mudaraddi* and K. B. Saxena ${ }^{1}$
}

Jawaharlal Nehru Technological University, Hyderabad, 500 085, Telangana; ${ }^{1}$ International Crops Research Institute for the Semi-Arid Tropics (ICRISAT), Patancheru 502 324, Telangana

(Received: June 2014; Revised: December 2014; Accepted: January 2015)

\begin{abstract}
A set of 155 hybrid parents was characterized at molecular level using 24 simple sequence repeat (SSR) markers. The number of alleles amplified ranged from 3 to 41 at an average of 14.5 alleles per marker and mean polymorphic information content (PIC) value of 0.64 . Based on genetic diversity, three heterotic groups were constituted. Male sterile (A) lines were distributed into two heterotic groups, while the fertility restorer $(R)$ lines formed three heterotic groups. Of the 135 restorers studied, $51(37.78 \%)$ were classified in heterotic group I, and $75(55.56 \%)$ in heterotic group II. Six interspecific derivatives and three other genotypes constituted the heterotic group III. These observations indicated the presence of limited genetic diversity among $A$ and $R$ lines. Based on the present results, it is recommended that for breeding high yielding pigeonpea hybrids, more attention be given to develop new genetically diverse hybrid parents.
\end{abstract}

Key words: Genetic diversity, heterotic group, pigeonpea, polymorphism, SSR markers

\section{Introduction}

Pigeonpea [Cajanus cajan (L.) Millsp.] is an important high protein pulse crop of Asia and Africa. Global efforts to breed high yielding cultivars in this crop never met the expected success and its productivity has remained low for the past 50 years. The genetic enhance of yield has therefore, become a major concern for pigeonpea scientists (Saxena 2008). The recent breakthrough in breeding a cytoplasmic nuclear male sterility (CMS) system [Saxena et al 2005] and natural out-crossing of the crop have provided an opportunity to pigeonpea breeders for breaking the decades-old low $(<800 \mathrm{~kg} / \mathrm{ha})$ yield plateau. In this context, recent release of the world's first pigeonpea hybrid (Saxena et al. 2013) with $46 \%$ yield advantage over traditional cultivars in farmers' fields, has given positive indications about the potential of hybrid technology in achieving significant productivity gains in the near future. The genetic diversity of parental lines is known to plays a significant role in breeding of high yielding hybrid cultivars. Therefore, its assessment among the potential parental genotypes has become an integral part of hybrid breeding programmes. The process of classifying parental lines on the basis of genetic diversity and their logical use in breeding hybrids has gradually evolved into the concept of 'heterotic groups'. Saxena and Sawargaonkar (2014) reported that in pigeonpea the heterosis for yield by crossing the lines representing diverse heterotic groups was higher as compared to those belonging to the same heterotic group. The success in any hybrid breeding programme primarily depends on the ability of breeders to select parents on the basis of genetic diversity and other related parameters. In this context, the use of molecular markers is the latest and most reliable technology because its results are not influenced by factors such as crop phenology, cropping system, environment or genotype $x$ environment interactions. In the present study, besides studying genetic diversity within 155 hybrid parents, their molecular data were also used to establish heterotic groups.

\section{Materials and methods}

\section{Plant materials and DNA extraction}

A total of 155 hybrid parents including 20 cytoplasmic nuclear male-sterile (A-) lines and 135 restorers were

*Corresponding author's e-mail: bharati_bth@yahoo.com

Published by the Indian Society of Genetics \& Plant Breeding, F2, First Floor, NASC Complex, PB\#11312, IARI, New Delhi 110012 Online management by indianjournals.com 
selected from ICRISAT's hybrid pigeonpea breeding programme for their molecular characterization. The genomic DNA for analysis was isolated from young leaves of three weeks old seedlings using the protocol (Cuc et al. 2008). The DNA quantity for each sample was determined on $0.8 \%$ agarose gel and DNA concentrations were normalized at $5 \mathrm{ng} / \mu \mathrm{l}$.

\section{SSR markers, polymerase chain reactions (PCRs) and electrophoresis}

For molecular characterization, a total of $24 \mathrm{M} 13$ labeled primer pairs were used (Bohra et al. 2012) and PCRs were performed in a $5 \mu \mathrm{l}$ reaction volume $[0.5 \mu \mathrm{l}$ of 10 $\times$ PCR buffer, $0.3 \mu \mathrm{l}$ of $25 \mathrm{mM} \mathrm{MgCl}_{2}, 0.5 \mu \mathrm{l}$ of 2 mMdNTPs, $0.15 \mu \mathrm{l}$ of $10 \mathrm{pM}$ primer (MWG-Biotech AG, Bangalore, India), $0.3 \mathrm{U}$ of Taq polymerase (Bioline, London, UK), and $1.0 \mu \mathrm{l}(5 \mathrm{ng})$ of template DNA] in 96-well microtitre plate (ABgene, Rockford, Illinois, USA) using thermal cycler Gene-Amp PCR System 9700 (Applied Biosystems, Foster City, California, USA). A touch-down PCR program was used to amplify DNA fragments. An initial denaturation for $3 \mathrm{~min}$ at $95^{\circ} \mathrm{C}$ was followed by initial 10 cycles of denaturation for $20 \mathrm{~s}$ at $94^{\circ} \mathrm{C}$, annealing for $20 \mathrm{~s}$ at $55^{\circ} \mathrm{C}$ (the annealing temperature for each cycle being reduced by $1^{\circ} \mathrm{C}$ per cycle), and extension for $30 \mathrm{~s}$ at $72^{\circ} \mathrm{C}$. Subsequently, 35 cycles of denaturation (20s at $94^{\circ} \mathrm{C}$ ), annealing $\left(20 \mathrm{~s}\right.$ at $\left.48^{\circ} \mathrm{C}\right)$, and extension (30s at $72^{\circ} \mathrm{C}$ ) were used, followed by final extension at $72^{\circ} \mathrm{C}$ for $20 \mathrm{~min}$. The PCR products were checked for ampli?cation on $1.2 \%$ agarose gel. The ampli?ed products were separated on capillary electrophoresis using ABI 3730 (Applied Biosystems, Foster City, CA, USA) and allele calling was performed using Gene Mapper software version 4.0 (Applied Biosystems, Foster City, CA, USA).

\section{Analysis of data}

The allelic data obtained in base pair (bp) were analyzed as allele size and these were used to prepare dendrogram using MEGA version 5.05 (Tamura et al 2011). The polymorphism information content (PIC) estimates of markers (referring to the value of a marker for detecting polymorphism within a given germplasm), was estimated according to Anderson et al. (1993). In the present study an attempt was also made to formulate heterotic groups with the limited number of SSR makers available.

\section{Results and discussion}

\section{Molecular diversity}

Marker polymorphism : All the SSR markers were found polymorphic for the pigeonpea genotypes, and these markers amplified a total of 347 alleles with an average of 14.5 alleles per marker (Table 1). The PIC values of the markers ranged from 0.13 (CcM1373) to 0.94 (CcM1011) with an average of 0.64. Among A lines, 134 alleles were amplified from 23 polymorphic SSR markers with an average value of 6 alleles per marker and a range of 2 (CcM0594) to 15 (CcM1366) alleles per marker. The PIC value among the male

Table1. Polymorphism across A and Rlines of pigeonpea

\begin{tabular}{|c|c|c|c|c|c|c|}
\hline \multirow{2}{*}{$\begin{array}{l}\text { SSR } \\
\text { markers }\end{array}$} & \multicolumn{2}{|c|}{ A lines } & \multicolumn{2}{|c|}{$\mathrm{R}$ lines } & \multicolumn{2}{|c|}{ Across all lines } \\
\hline & $\begin{array}{l}\text { No. of } \\
\text { alleles }\end{array}$ & $\begin{array}{l}\text { PIC } \\
\text { value }\end{array}$ & $\begin{array}{l}\text { No. of } \\
\text { alleles }\end{array}$ & $\begin{array}{l}\text { PIC } \\
\text { value }\end{array}$ & $\begin{array}{l}\text { No. of } \\
\text { alleles }\end{array}$ & $\begin{array}{l}\text { PIC } \\
\text { value }\end{array}$ \\
\hline CcM1373 & 1 & 0 & 5 & 0.16 & 7 & 0.13 \\
\hline CcM0594 & 2 & 0.22 & 5 & 0.28 & 5 & 0.28 \\
\hline CcM2409 & 3 & 0.41 & 3 & 0.36 & 3 & 0.34 \\
\hline CcM2505 & 3 & 0.47 & 3 & 0.37 & 3 & 0.39 \\
\hline CcM0962 & 3 & 0.35 & 5 & 0.38 & 7 & 0.44 \\
\hline CcM1079 & 5 & 0.40 & 9 & 0.41 & 10 & 0.45 \\
\hline CcM0785 & 4 & 0.33 & 8 & 0.6 & 10 & 0.56 \\
\hline CcM2221 & 3 & 0.56 & 5 & 0.52 & 9 & 0.57 \\
\hline CcM2379 & 5 & 0.34 & 8 & 0.62 & 9 & 0.57 \\
\hline CcM0673 & 3 & 0.53 & 5 & 0.58 & 7 & 0.60 \\
\hline CcM2818 & 5 & 0.62 & 12 & 0.6 & 12 & 0.63 \\
\hline CcM2241 & 4 & 0.53 & 6 & 0.66 & 7 & 0.66 \\
\hline CcM2332 & 8 & 0.79 & 10 & 0.70 & 13 & 0.75 \\
\hline CcM2697 & 6 & 0.71 & 13 & 0.75 & 13 & 0.76 \\
\hline CcM1207 & 4 & 0.58 & 18 & 0.80 & 21 & 0.77 \\
\hline CcM1109 & 7 & 0.73 & 11 & 0.75 & 13 & 0.78 \\
\hline CcM2871 & 9 & 0.82 & 18 & 0.76 & 21 & 0.78 \\
\hline CcM2710 & 7 & 0.70 & 15 & 0.75 & 19 & 0.79 \\
\hline CcM1982 & 6 & 0.71 & 9 & 0.74 & 11 & 0.79 \\
\hline CcM0988 & 6 & 0.74 & 12 & 0.75 & 13 & 0.81 \\
\hline CcM2895 & 6 & 0.75 & 24 & 0.88 & 28 & 0.87 \\
\hline CcM0443 & 8 & 0.79 & 22 & 0.89 & 28 & 0.88 \\
\hline CcM1366 & 15 & 0.9 & 28 & 0.91 & 37 & 0.91 \\
\hline CcM1011 & 11 & 0.82 & 34 & 0.94 & 41 & 0.94 \\
\hline Maximum & 15 & 0.9 & 34 & 0.94 & 41 & 0.94 \\
\hline Minimum & 1 & 0 & 3 & 0.16 & 3 & 0.13 \\
\hline Mean & 6 & 0.57 & 12 & 0.63 & 14.5 & 0.64 \\
\hline
\end{tabular}


sterile $(A)$ lines ranged from 0.22 (CcM0594) to 0.90 (CcM1366) with an average of 0.57 . Similarly, among the restorer lines, a total of 288 alleles were amplified from the polymorphic SSR markers with an average of 12 alleles per marker and range from 3 (CcM2409 and CcM2505) to 34 (CcM1011) alleles per marker. The PIC values for this group of materials ranged from 0.16 (CcM1373) to 0.94 (CcM1011) with an average of 0.63 .

\section{Marker variability}

To assess the molecular diversity among the test materials, UPGMA - based tree was constructed using the marker genotyping data. The dendrogram (Fig. 1) revealed limited molecular diversity among the genotypes with three distinct clusters. A perusal of data further revealed that Cluster I had all the early maturing and a few medium maturing materials. Cluster Il comprised of only medium maturing genotypes. Cluster III was distinct and included three germplasm and six inter-specific derivatives involving three wild relatives viz., C. acutifolius, $C$. scarabaeoides and $C$. platycarpus of pigeonpea. The results showed that at molecular level this group of inter-specific derivatives was the most diverse lot. The present study also confirmed the earlier observations (Burns et al. 2001; Panguluri et al. 2006; Odeny et al. 2007; Yang et al 2006; Saxena et al. 2010a, 2010b) that the cultivated pigeonpea suffers from inherent limited molecular diversity.

\section{Heterotic groups}

Heterotic groups of male sterile lines

Twenty male sterile (A) lines were classified into two heterotic groups, suggesting relatively less nuclear diversity. Among these, eight $A$ lines found place in heterotic group I, and these involved five early maturing [ICPA 2188, ICPA 2156, ICPA 2078, ICPA 2089, ICPA 2039] and three [ICPA 2043, ICPA 2170, ICPA 2042] medium maturing types. The remaining $12 \mathrm{~A}$ lines (Table 2) were placed in heterotic group II, and all of them were of medium maturity group. This classification showed that in the early maturing group the choice of selecting $A$ lines on the basis of genetic diversity is limited. In the medium maturing group however, out of $15 \mathrm{~A}$ lines studied, three were placed in heterotic group I and the remaining 12 in heterotic group II. These results suggested that at present the genetic diversity among $\mathrm{A}$ lines is not sufficient for a dynamic hybrid pigeonpea breeding programme; hence there is an urgent need to diversify the genetic base of the female parents.
Heterotic groups of fertility restoring lines

A total of 135 fertility restoring lines were studied and this group exhibited a large phenotypic variation with respect to different agronomy traits (Saxena et al. 2014) but in the present study the variability at molecular level was found limited. From this material three heterotic groups were constructed. Heterotic group I had $51(37.78 \%)$ lines, while heterotic group II included $75(55.56 \%)$ lines. Only nine $(6.67 \%)$ fertility restorers constituted the heterotic group III (Table 2). This group had useful variability since it included six inter-specific derivatives, representing three wild relatives of pigeonpea. ICPL 20343, ICPL 20346 and ICPL 20347 were derived from C. acutifolius; ICPL 20342 from C. scarabaeoides; and ICPL 20348 and ICPL 20349 from C. platycarpus. The former two wild species represent secondary gene pool, while $C$. platycarpus is classified in the tertiary gene pool of genus Cajanus. The remaining genotypes were ICPL 87119 and its two selections. The present results also showed that majority $(93.34 \%)$ of the fertility restoring lines were placed in the first two heterotic groups, suggesting limited genetic variability among this group of genotypes also. The six inter-specific derivatives may be useful in breeding new male parental lines.

The utility of geographic diversity in hybrid maize breeding was recognized about a hundred year back, but today it may not stand alone because of rapid movement of cultivars across geographical boundaries and gradual replacement of landraces with modern high yielding cultivars. In this situation the relevance of genetic diversity among parents becomes more important in breeding hybrids. To identify genetically diverse genotypes, the crop breeders traditionally use different statistical tools to separate environmental effects from the total recognizable phenotypic variability. In maize and other important crops, these technologies have been frequently used with encouraging results. With the recent development of sophisticated genomic techniques, the environment component of variation can now be totally eliminated and reliable information on the genetic diversity of the parents can be generated for use in the formation of heterotic pools and breeding hybrids.

As mentioned earlier, the stagnation of pigeonpea productivity has been a subject of concern for a long time and the evolution of hybrid technology has given good signals towards breaking the yield plateau in this crop (Saxena 2008). The success of this programme, however, will depend on the availability 
Table 2. Heterotic groups of A- and R- lines based on molecular diversity

\begin{tabular}{|c|c|c|c|}
\hline & Heterotic Group I & Heterotic Group II & Heterotic Group III \\
\hline A-lines & $\begin{array}{l}\text { ICPA 2188, 2156, 2078, } \\
2043,2170,2042,2089,2039\end{array}$ & $\begin{array}{l}\text { ICPA 2201, 2048, 2204, 2207, 2208, 2092, 2098, } \\
\text { 2202, 2047, 2189, 2050, } 2051\end{array}$ & \\
\hline \multirow[t]{2}{*}{ R-lines } & $\begin{array}{l}\text { BDN-1, MN5, JBP 36B, Sarita, } \\
\text { Pusa Ageti, UPAS 120, Pragati }\end{array}$ & $\begin{array}{l}\text { ICPL 87091, 99009, 99010,99015, 94062, 20130, } \\
20137,151,20126,20103,20139,20098,96061, \\
20094,20205,20203,20129,20123,149,88039, \\
20101,20092,99008,99013,20096,20116,20134, \\
20132,20131,20113,20138,20236,331,99054,81, \\
88034,88038,86022,20242,20095,20119,20129, \\
20122,20204\end{array}$ & ICPR 2671, 2740 \\
\hline & $\begin{array}{l}\text { ICPX060137, ICPX060148, } \\
\text { ICPX060134, ICPX060138, } \\
\text { ICPX060142, ICPX040146, } \\
\text { ICP 2376, ICP 7035, ICP 5529, } \\
\text { JBP110B, MAL 9, MAL 12, } \\
\text { MAL 15, Lakshmi, Kanchan, } \\
\text { Vaishali }\end{array}$ & $\begin{array}{l}\text { ICPL 87, 161, 131, 20107, 87053, 20097, 20127, } \\
20177,99005,20237,20100,85063,20124, \\
20125,20115,20117,99051,99050,20241, \\
20112,20106,20120,20109,20186,20202, \\
20099,20243,99055,96053,20238,99061, \\
99004,83057,99046,20108,20136,332,20104, \\
20102,96058,87051,20114,20110,20058,20093 \\
20120,99044,20128,20135,20105,20111,20133, \\
20201,20121,20187,99048,20187,20188,20118\end{array}$ & $\begin{array}{l}\text { ICPL 87119, 20343, } \\
20342,20346, \\
20347,20348, \\
20349\end{array}$ \\
\hline
\end{tabular}

of productive hybrid parents with large genetic diversity for per se performance, greater combining ability and important market-preferred traits. In this context evaluation, selection, and classification of germplasm into different heterotic groups will be of great help in breeding hybrids. At this point it is also to be noted that there is no single rule to constitute heterotic groups in any crop. Reif et al. (2003), while summarizing the subject, concluded that the classification of germplasm into heterotic groups can be done on the basis of per se performance of hybrids, combining ability of hybrids, origin of the parents or their genetic diversity. The information generated on phenotypic diversity using different statistical tools cannot be used effectively for developing heterotic groups as the data are undoubtly influenced by different environmental factors and their variable interactions with different genotypes.

Fan et al. (2003) and Jelena et al. (2007), therefore, advocated that judicious use of pedigree information (origin), combining ability, and molecular markers together may be the most effective way to develop heterotic groups. Use of genomic approach in constituting heterotic groups has a great significance in hybrid pigeonpea breeding. This is because in this crop there are some "super traits', which when present / expressed, change the entire phenotype of a plant, and hence the expression of yield and yield components is significantly altered. In such situations the morphological crop data will not represent real genotypic variability. The best example in this respect is the effect photo-period on plant phenology. Pigeonpea is known to be highly sensitive to photoperiod and require short days for the induction of flowering (Saxena 2008). In order to fetch good harvest, the sowing of commercial pigeonpea crop is done during long days and it accumulates a considerable biomass before it flowers at the onset of short days in 100-120 days. Under this crop culture each plant grows over two meter tall and produces large biomass with $>500$ pods. Alternatively, if the same genotype is planted at the onset of short days in the month of September, then the plants will flower in 60-65 days and will attain height of 70-75 cm and produce only about 50 pods/plant. The other such traits which influence the phenology of pigeonpea plants are growth habit (determinate versus non-determinate), temperature, and cultivar maturity (early versus late). Thus, the data on same genotype in the two environments will be markedly different, and real picture about their variability will never emerge. Therefore for assessing genetic variability among genotypes with accuracy, the use of genomics data is the best bet to have real picture about the diversity of the genotypes.

In pigeonpea so far only one report (Saxena and Sawargaonkar 2014) has been published on the development and use of heterotic groups. The authors in this case formulated seven heterotic groups based on specific combining ability effects estimated in a 
set of line $x$ tester crosses, evaluated at four diverse locations. The present exercise is the first of its kind in pigeonpea, where a set of molecular data has been used to generate information on heterotic groups. The diversity among hybrid parents based on morphological data was large (Saxena et al. 2014), but these observations were not supported at molecular level. Low genetic variability among the cultivated pigeonpea was also reported earlier (Burns et al. 2001; Panguluri et al. 2006; Odeny et al. 2007; Yang et al. 2006; Saxena et al. 2010a, 2010b) it may limit the hybrid gains in the crop. It is therefore, recommended that for developing high yielding hybrids in pigeonpea, a dynamic breeding programme aimed to enhance the genetic variability among hybrid parents should be launched and given a very high priority. The presence of heterotic group III, where six lines were derived from inter-specific crosses, provided a silver lining. The question of limited genetic diversity within primary gene pool can be adequately addressed by utilizing wild relatives of pigeonpea. There are 32 wild relatives of pigeonpea which can provide plenty of genetic diversity to hybrid breeding programmes.

\section{Acknowledgements}

The authors acknowledge the help of Genotyping Services Laboratory (GSL) of ICRISAT. The financial support received from Bill and Melinda Gates Foundation (TL II Project) is also acknowledged.

\section{References}

Anderson J. A., Churchill G. A., Sutrique J. E., Tanksley S. D. and Sorrells M. E. 1993. Optimizing parental selection for genetic linkage maps. Genome, 36: 181-186.

Bohra A., Saxena R. K., Gnanesh B. N., Saxena K. B., Byregowda M., Rathore A., Kavikishor P.B., Cook D. R. and Varshney R. K. 2012. An intra-specific consensus genetic map of pigeonpea [Cajanus cajan (L.) Millsp.] derived from six mapping populations. Theor. Appl. Genet., 25(6):1325-38.

Burns M. J., Edwards K. J., Newbury H. J., Ford-Lloyd B. V. and Baggott C. D. 2001. Development of simple sequence repeat (SSR) markers for the assessment of gene flow and genetic diversity in pigeonpea (Cajanus cajan). Mol. Ecology Notes 1:283-285

Cuc L. M., Mace E. S., Crouch J. H., Quang V. D., Long T. D. and Varshney R. K. 2008 Isolation and characterization of novel microsatellite markers and their application for diversity assessment in cultivated groundnut (Arachis hypogaea). BMC Plant Biology, 8: 55.

Fan X. M., Tan J. Y., Zhang M. S., Li Y. X., Huang Y. X., Yang J.Y., Peng Z. B. and Li X. H. 2003. Heterotic grouping for 25 tropical maize inbreds and 4 temperate maize inbreds by SSR markers. Acta Sin., 29: $835-840$.

Jelena S., Snezana P., Zorica M. D. and Milomir F. 2007. Characterization of maize inbred lines based on molecular markers, heterosis and pedigree data. Genetika, 39: 355-363.

Odeny D. A., Jayashree B., Ferguson M., Hoisington D., Crouch J. and Gebhardt C. 2007. Development, characterization and utilization of microsatellite markers in pigeonpea. Plant Breed., 126: 130-137.

Panguluri S. K., Janaiah K., Govil J. N., Kumar P. A. and Sharma P. C. 2006. AFLP fingerprinting in pigeonpea (Cajanus cajan (L.) Millsp.) and its wild relatives. Genetic Resou. Crop Evol., 53: 523-531.

Reif J. C., Melchinger A. E., Xia X. C., Warburton M. L., Hoishington D. A., Vasal S. K., Bohn M. and Frisch M. 2003. Use of SSRs for establishing heterotic groups in sub tropical maize. Theor. Appl. Genet., 107: $947-957$

Saxena K. B. 2008. Genetic improvement of pigeonpeaa review. Tropical Plant Biology, 1: 159-178.

Saxena K. B. and Sawargaonkar S. L. 2014. First information on heterotic groups in pigeonpea [Cajanus cajan (L.) Millsp.]. Euphytica, doi: 10.1007/ s10681-014-1142-0

Saxena K. B., Kumar R. V. and Bharathi M. 2014. Studies on fertility restoration of $\mathrm{A} 4$ cytoplasm in pigeonpea. Euphytica, 198(1): 127-135.

Saxena K. B., Kumar R. V., Srivastava N. and Shiying B. 2005. A cytoplasmic-nuclear male-sterility system derived from a cross between Cajanus cajanifolius and Cajanus cajan. Euphytica, 145: 289-294.

Saxena K. B., Kumar R. V., Tikle A. N. et al. 2013. ICPH 2671 - The world's first commercial food legume hybrid. Plant Breed., 132(5): 479-485.

Saxena R. K., Prathima C., Saxena K. B., Hoisington D. A., Singh N. K. and Varshney R. K. 2010a. Novel SSR markers for polymorphism detection in pigeonpea (Cajanus spp.). Plant Breed., 129: 142-148.

Saxena R. K., Saxena K. B., Kumar R. V., Hoisington D. A. and Varshney R. K. 2010b. SSR-based diversity in elite pigeonpea genotypes for developing mapping populations to map resistance to Fusarium wilt and sterility mosaic disease. Plant Breed., 129:135-141.

Tamura K., Peterson D., Peterson N., Stecher G., Nei M. and Kumar S. 2011. MEGA5: Molecular evolutionary genetics analysis using maximum likelihood, evolutionary distance, and maximum parsimony methods. Mol. Biology Evol., 28: 2731-2739.

Yang S., Pang W., Ash G., Harper J., Carling J., Wenzl P., Huttner E., Zong X. and Kilian A. 2006. Low level of genetic diversity in cultivated pigeonpea compared to its wild relatives is revealed by diversity arrays technology. Theor. Appl. Genet., 113: 585-595. 